\title{
Root Asymptotics for the Eigenfunctions of Univariate Differential Operators
}

\author{
B. Shapiro
}

\begin{abstract}
This paper is a brief survey of the research conducted by the author and his collaborators in the field of root asymptotics of (mostly polynomial) eigenfunctions of linear univariate differential operators with polynomial coefficients.
\end{abstract}

Keywords: root-counting measure, exactly solvable operator, Schrödinger equation.

\section{Objective}

Study asymptotic properties of sequences $\left\{p_{n}(z)\right\}$, of polynomials/entire functions in $z$ which either

1. are polynomial/entire eigenfunctions of a univariate linear ordinary differential operator with polynomial coefficients;

or

2. are polynomial solutions of more general pencils of such operators, e.g. homogenized spectral problems and Heine-Stieltjes spectral problems; or

3. satisfy a finite recurrence relation with (in general) varying coefficients.

\section{Basic notions and examples}

Definition 1 An operator $T=\sum_{i=1}^{k} Q_{i}(z) \frac{d^{i}}{d z^{i}}$ is called exactly solvable if $\operatorname{deg} Q_{i}(z) \leq i$ and there exists at least one value $i$ such that $\operatorname{deg} Q_{i}(z)=i$.

Obviously, $T\left(z^{j}\right)=a_{j} z^{j}+$ lower order terms, i.e. $T$ acts by an (infinite) triangular matrix in the monomial basis $\left\{1, z,, z^{2}, \ldots\right\}$ of $\mathbb{C}[z]$.

Lemma 1 For any exactly solvable $T$ and sufficiently large $n$ there exists a unique (up to a scalar) eigenpolynomial $p_{n}(z)$ of degree $n$.

Typical problem. Given an exactly solvable $T$ describe the root asymptotics for the sequence of polynomials $\left\{p_{n}(z)\right\}$.

\subsection{Two asymptotic measures}

Given a polynomial family $\left\{p_{n}(z)\right\}$ where $\operatorname{deg} p_{n}(z)=n$ we define two basic measures: (i) asymptotic root-counting measure $\mu$; (ii) asymptotic ratio measure $\nu$.
Definition 2 Associate to each $p_{n}(x)$ a finite probability measure $\mu_{n}$ by placing the mass $\frac{1}{n}$ at every root of $p_{n}(x)$. (If some root is multiple we place at this point the mass equal to its multiplicity divided by n.) The limit $\mu=\lim _{n} \mu_{n}$ (if it exists in the sense of weak convergence) will be called the asymptotic root-counting measure of $\left\{p_{n}(z)\right\}$.

Definition 3 Consider the ratio $q_{n}(z)=\frac{p_{n-1}(z)}{p_{n}(z)}$. (Assume for simplicity that $p_{n}(z)$ has no multiple roots and expand $q_{n}(z)=\sum_{i=1}^{n} \frac{\kappa_{i, n}}{z-z_{i, n}}$.) Associate to $q_{n}(z)$ the finite complex-valued measure by placing $\kappa_{i, n}$ at $z_{i, n}$. Define the asymptotic ratio measure of the sequence $\left\{p_{n}(z)\right\}$ as

$$
\nu=\lim _{n \rightarrow \infty} \nu_{n} \text {. }
$$

Observation. Supports of $\mu$ and $\nu$ coincide but $\nu$ is often complex-valued.

\section{$2.2 \quad$ Examples}

Below we show the root distribution for $p_{55}(z)$ for 4 different exactly solvable operators

$$
\begin{aligned}
T_{1}= & z(z-1)(z-I) \frac{d^{3}}{d z^{3}} \\
T_{2}= & (z-I)(z+I)(z-2+3 I)(z-3-2 I) \frac{d^{4}}{d z^{4}} \\
T_{3}= & (z-I)(z+I)(z-2+3 I)(z-3-2 I) \\
& (z+3) \frac{d^{5}}{d z^{5}} ; \\
T_{4}= & \left(z^{2}+1\right)(z-2+3 I)(z-3-2 I)(z+3) \\
& (z+1+I) \frac{d^{6}}{d z^{6}}
\end{aligned}
$$

of the form $Q(z) \frac{d^{k}}{d z^{k}}$ where $Q(z)$ is a monic polynomial of degree $k+1$. 

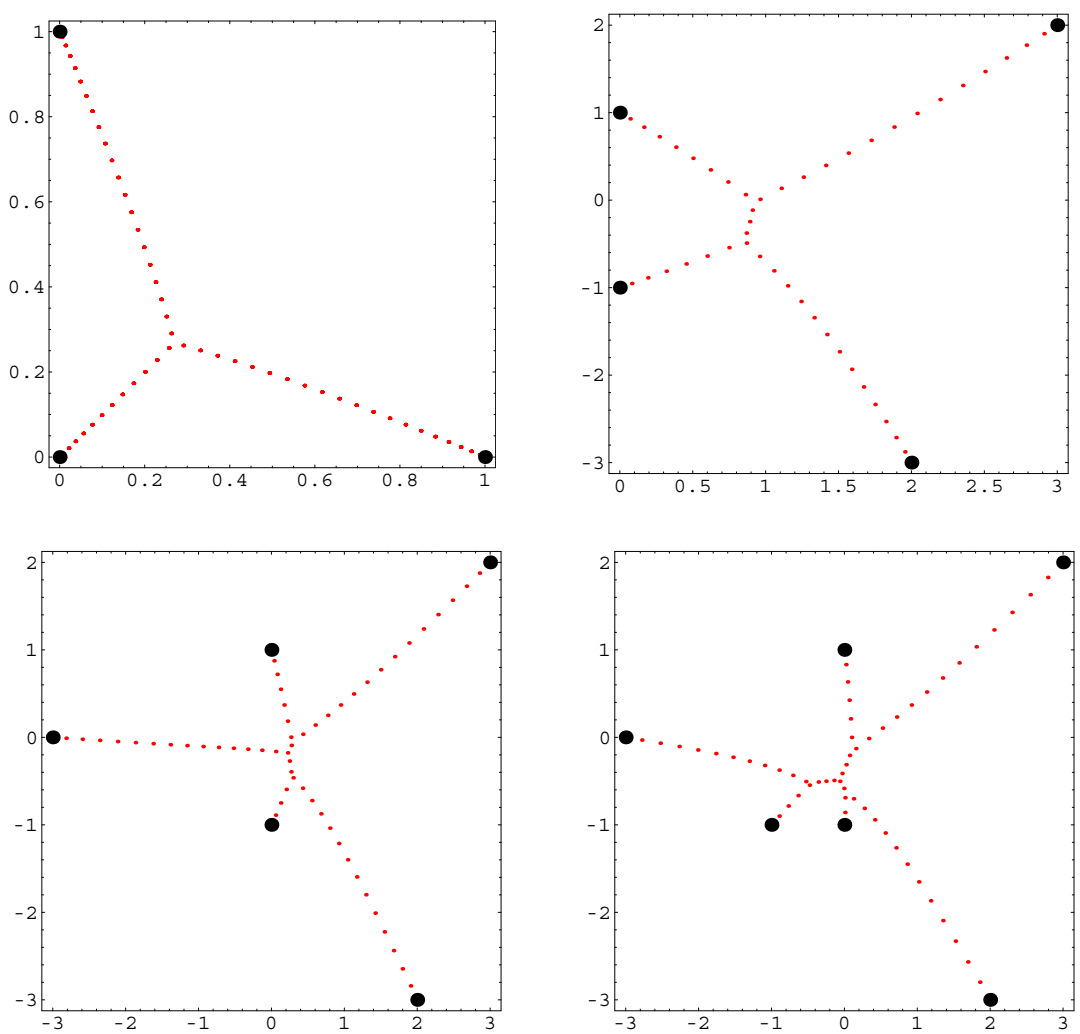

Fig. 1: Roots of $p_{55}(z)$ for the above $T$ 's

Explanations to Fig. 1. The larger dots show the roots of the corresponding $Q(z)$ and the smaller dots are the fifty five roots of the corresponding $p_{55}(z)$.

\subsection{Classical prototypes}

Theorem 1 (G. Szegö) If $\left\{p_{n}(z)\right\}$ is a family of polynomials orthogonal w.r.t a positive weight $w(z)$ supported on $[-1,1]$ such that $\int_{-1}^{1} \ln w(z) \mathrm{d} z<\infty$ then the asymptotic root-counting measure has the density $\frac{1}{\pi \sqrt{1-z^{2}}}, x \in[-1,1]$.

Theorem 2 (G. Szegö) If $\left\{p_{n}(z)\right\}$ is a family of polynomials orthogonal w.r.t a weight $w(z)$ supported on $[-1,1]$ such that $\int_{-1}^{1} \frac{\ln w(z) \mathrm{d} z}{\sqrt{1-z^{2}}}>-\infty$ then the asymptotic ratio measure has the density $\frac{2 \sqrt{1-z^{2}}}{\pi}, z \in[-1,1]$

\section{First results}

\subsection{Non-degenerate exactly solvable operators}

The next subsection is based on $[10,2]$.

Definition 4 The Cauchy transform of a (com- plex-valued) measure $\rho$ satisfying $\int_{\mathbb{C}} \mathrm{d} \rho(\xi)<\infty$ is given by

$$
C_{\rho}(z)=\int_{\mathbb{C}} \frac{\mathrm{d} \rho(\xi)}{z-\xi}
$$

Example. If $\rho(z)=\frac{1}{\pi \sqrt{1-z^{2}}}, z \in[-1,1]$ then $C_{\mu}=\frac{1}{\sqrt{z^{2}-1}}$ in $\mathbb{C} \backslash[-1,1]$ and $C_{\nu}=\frac{2}{z+\sqrt{z^{2}-1}}$ in $\mathbb{C} \backslash[-1,1]$.

Definition $5 \quad$ An exactly solvable operator $T=\sum_{i=1}^{k} Q_{i}(z) \frac{d^{i}}{d z^{i}}$ is called non-degenerate if $\operatorname{deg} Q_{k}(z)=k$.

Proposition 1 Assuming that $\Psi(z)=\lim _{n \rightarrow \infty} \frac{p_{n}^{\prime}(z)}{n p_{n}(z)}$ exists in some open neighborhood $\Omega$ of $\mathbb{C}$ one gets that $\Psi(z)$ satisfies in $\Omega$ the algebraic equation

$$
Q_{k}(z) \Psi^{k}(z)=1
$$

Theorem 3 (H. Rullgård) Let $Q_{k}(z)$ be a monic degree $k$ polynomial. Then there exists a unique probability measure $\mu_{Q}$ such that

1) $\operatorname{supp} \mu_{Q}$ is compact;

2) its Cauchy transform $C_{\mu}$ satisfies the equation $Q_{k}(z) C_{\mu}^{k}(z)=1$ almost everywhere in $\mathbb{C}$. 

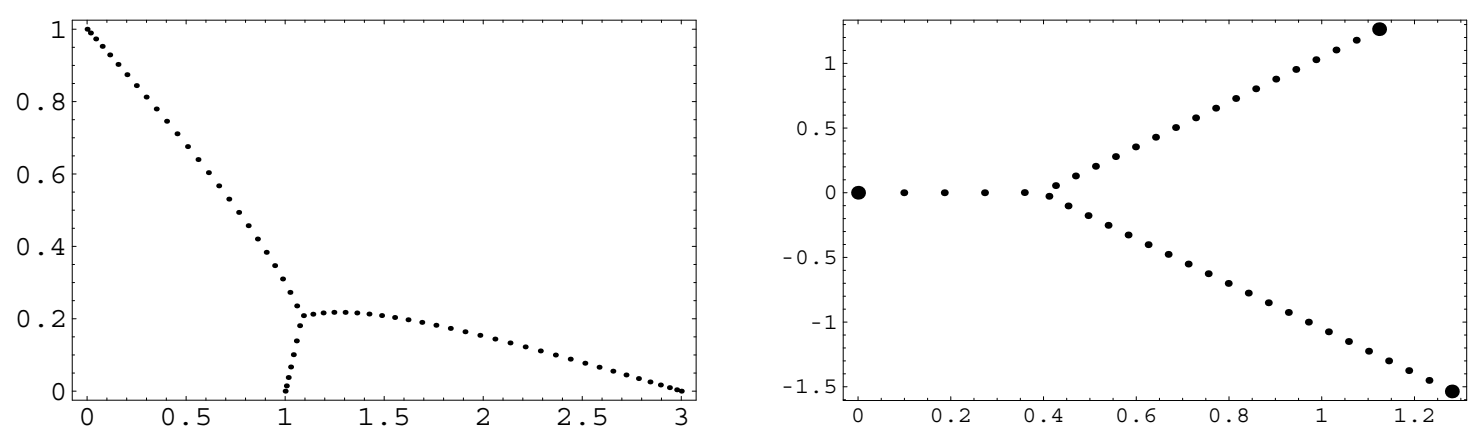

Fig. 2: The measure $\mu_{Q}$ before and after the straightening transformation in the case $Q(z)=(z-1)(z-3)(z-I)$

Theorem 4 (Main result, see Fig. 2) In the above notation

1) $\operatorname{supp} \mu_{Q}$ is a curvilinear tree which is straightened out by the analytic mapping

$$
\xi(z)=\int_{a}^{z} \frac{\mathrm{d} z}{\sqrt[k]{Q_{k}(z)}} .
$$

2) $\operatorname{supp} \mu_{Q}$ contains all the zeros of $Q_{k}(z)$ and is contained in the convex hull of those.

3) There is a natural formula for the angles between the branches, and the masses of the branches satisfy Kirchhoff law.

Below we show an example of such a measure in a proper scale and with all angles between its vertices marked, see Fig. 3.

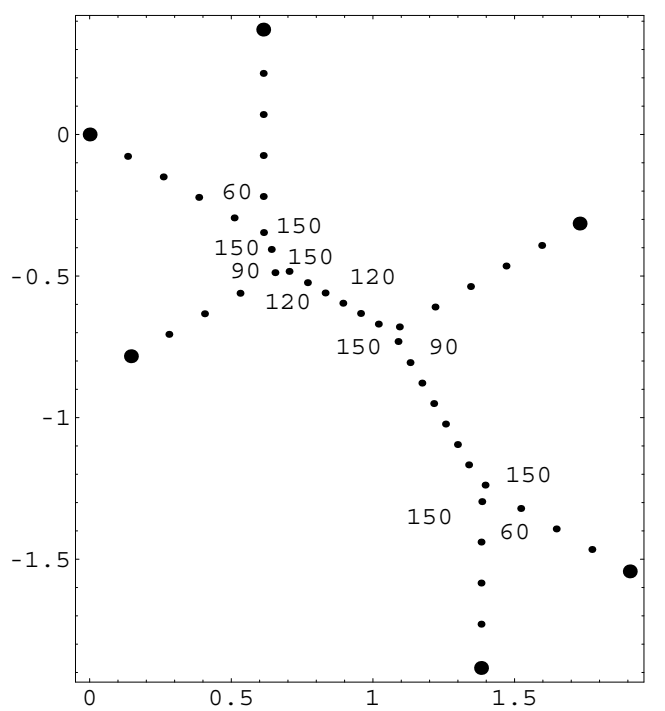

Fig. 3: Example of $\mu_{Q}$ with angles

Problem 1 Is it true that the support of the measure $\mu_{Q}$ is a subset of the Stokes lines of the corresponding operator $Q \frac{d^{k}}{d z^{k}}$ ?

Some partial results in this direction can be found in $[12]$.

\subsection{Degenerate exactly solvable operators}

This subsection is based on [1].

Definition 6 An exactly solvable $T$ of order $k$ is called degenerate iff $\operatorname{deg} Q_{k}<k$.

Classical examples: $T=z \frac{d^{2}}{d z^{2}}+(a z+b) \frac{d}{d z}, T=$ $\frac{d^{2}}{d z^{2}}+(a z+b) \frac{d}{d z}$ leading to Laguerre resp. Hermite polynomials.

Proposition 2 The union of all roots of all polynomial eigenfunctions of an exactly solvable $T$ is unbounded if and only if $T$ is degenerate.

Problem 2 Given a degenerate $T$ with the family of eigenpolynomials $\left\{p_{n}(z)\right\}$ how fast does the maximum $r_{n}$ of the modulus of roots of $p_{n}(z)$ grow?

Conjecture 1 Given a degenerate $T=$ $\sum_{j=1}^{k} Q_{j}(z) \frac{d^{j}}{d z^{j}}$ denote by $j_{0}$ the largest $j$ for which $\operatorname{deg} Q_{j}(z)=j$. Then

$$
\lim _{n \rightarrow \infty} \frac{r_{n}}{n^{d}}=c_{T}
$$

where $c_{T}>0$ is a positive constant and

$$
d:=\max _{j \in\left[j_{0}+1, k\right]}\left(\frac{j-j_{0}}{j-\operatorname{deg} Q_{j}}\right) .
$$

\section{Corollary 1 (of the latter Conjecture)}

The Cauchy transform $C(z)$ of the asymptotic root measure $\mu$ of the scaled eigenpolynomial $q_{n}(z)=$ $p_{n}\left(n^{d} z\right)$ of a degenerate $T$ satisfies the following algebraic equation for almost all complex $z$ :

$$
z^{j_{0}} C^{j_{0}}(z)+\sum_{j \in A} \alpha_{j, \operatorname{deg} Q_{j}} z^{\operatorname{deg} Q_{j}} C^{j}(z)=1,
$$

where $A$ is the set consisting of all $j$ for which the maximum $d:=\max _{j \in\left[j_{0}+1, k\right]}\left(\frac{j-j_{0}}{j-\operatorname{deg} Q_{j}}\right)$ is attained, i.e. $A=\left\{j:\left(j-j_{0}\right) /\left(j-\operatorname{deg} Q_{j}\right)=d\right\}$. 

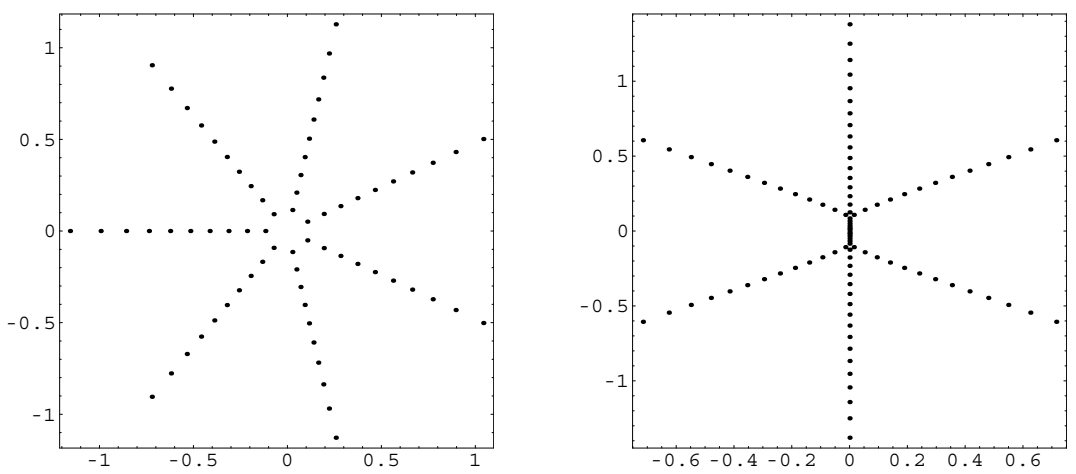

Fig. 4: Examples of the root distributions of scaled eigenpolynomials to degenerate exactly solvable operators

The latter equation for the Cauchy transform (if true) leads to very detailed information about the support of the asymptotic root-counting measure for the sequence of scaled eigenpolynomials. We illustrate this in Fig. 4.

\section{Homogenized spectral problem for non-degenerate $T$}

This section is based on [6]. An observant reader has noticed that so far only the leading coefficient of an exactly solvable operator effected the asymptotic root-counting measure, which makes the situation somewhat unsatisfactory.

To make the whole symbol of an operator important we consider (following the classical pattern of e.g. W. Wasow, M. Fedoryuk) the homogenized spectral problem of the form

$$
T_{\lambda}=\sum_{i=0}^{k} Q_{i}(z) \lambda^{k-i} \frac{d^{i}}{d z^{i}}
$$

where each $Q_{i}(x)=a_{i i} z^{i}+a_{i, i-1} z^{i-1}+\ldots$ is a polynomial of degree $i$.

Definition 7 A non-degenerate $T$ is called of general type iff $\operatorname{deg} Q_{k}(z)=k$ and $\sum_{i=0}^{k} a_{i i} \lambda^{k-i}=0$ has $k$ distinct zeros.

Proposition 3 If $T$ is of general type then

1) for all sufficiently large $n$ there exist exactly $k$ distinct values $\lambda_{n, j}, j=1, \ldots, k$ of the spectral parameter $\lambda$ such that the operator $T_{\lambda}$ has a polynomial eigenfunction $p_{n, j}(z)$ of degree $n$.

2) Asymptotically $\lambda_{n, j} \sim n \lambda_{j}$ where $\lambda_{1}, \ldots, \lambda_{k}$ is the set of roots of the algebraic equation $\sum_{i=0}^{k} a_{i, i} x^{k-i}=0$.
Conjecture 2 If $T$ is of general type and all $\lambda_{1}, \ldots, \lambda_{k}$ have distinct arguments then for each $j=$ $1, \ldots, k \exists$ ! probability measure $\mu_{j}$ with compact support whose Cauchy transform $C_{j}(z)$ satisfies almost everywhere in $\mathbb{C}$

$$
\sum_{i=1}^{k} Q_{i}(z)\left(\lambda_{j} C_{j}(z)\right)^{i}=0
$$

Conjecture $3 C_{j}(z)=\lim _{n \rightarrow \infty} \frac{p_{n, j}^{\prime}(z)}{\lambda_{n, j} p_{n, j}(z)}$ outside the support of $\mu_{j}$ which is the union of finitely many segments of analytic curves forming a curvilinear tree.

Observation. Near $\infty \in \mathbb{C P}^{1}$ the Cauchy transforms $\lambda_{1} C_{1}(z), \ldots, \lambda_{k} C_{k}(z)$ are independent sections of the symbol equation of $T_{\lambda}$ considered as a branched cover over $\mathbb{C P}^{1}$.

Problem 3 Find an explicit description of (the support) of the measures $\mu_{i}$. Is there any relation of these measures to the periods of the plane curve $\sum_{i=1}^{k} Q_{i}(z) y^{i}=0$ ?

\section{Heine-Stieltjes theory}

This section is based on [11]. Take an arbitrary univariate linear differential operator $T=\sum_{i=0}^{k} Q_{i}(z) \frac{d^{i}}{d z^{i}}$ with polynomial coefficients and set

$$
r=\max _{i}\left(\operatorname{deg} Q_{i}(z)-i\right)
$$

Definition 8 If $r \geq 0, \operatorname{deg} Q_{k}(z)=k+r$ and $Q_{k}(z)$ has at least two distinct roots we call $T$ a general Lame-type operator. 

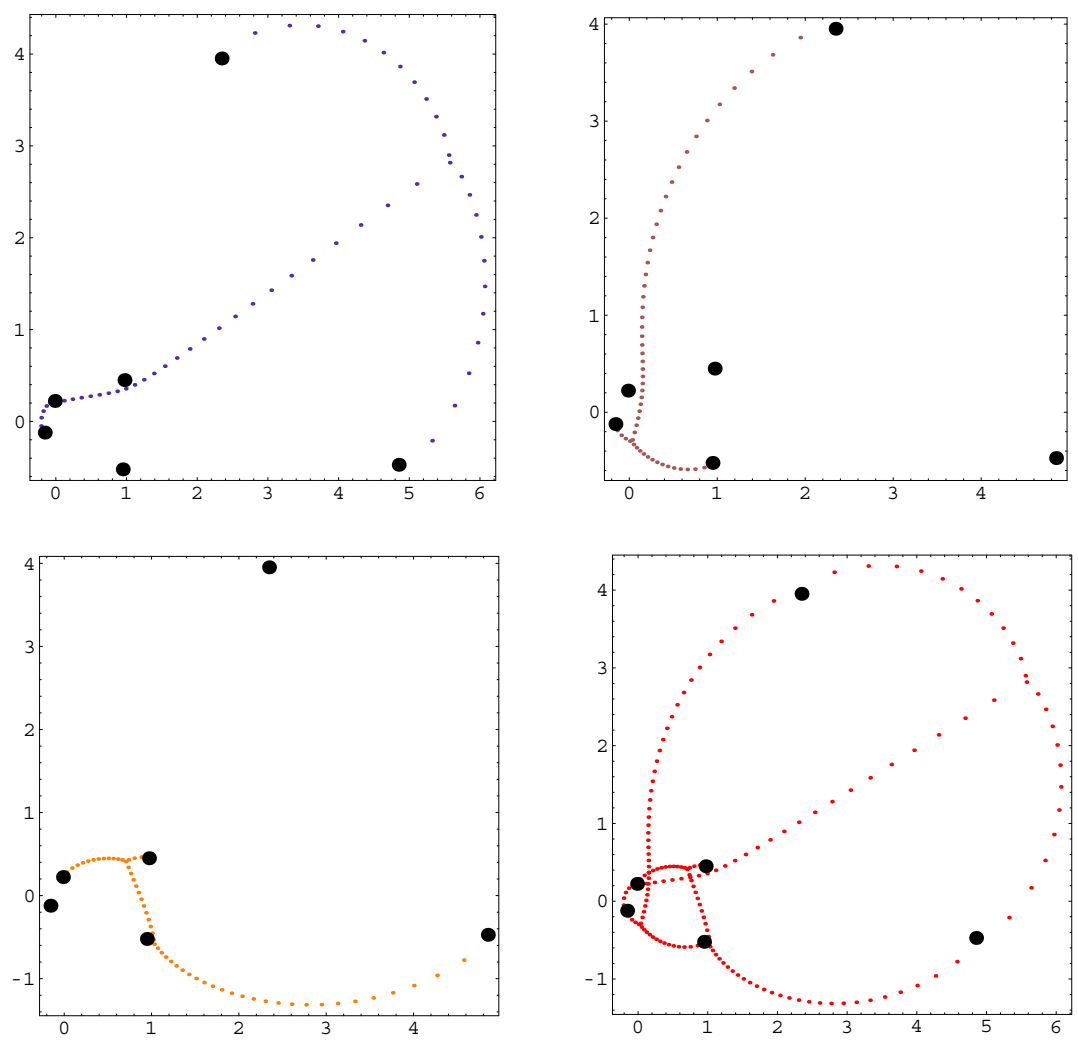

Fig. 5: Three root-counting measures and their union for a homogenized spectral problem with an operator of order 3
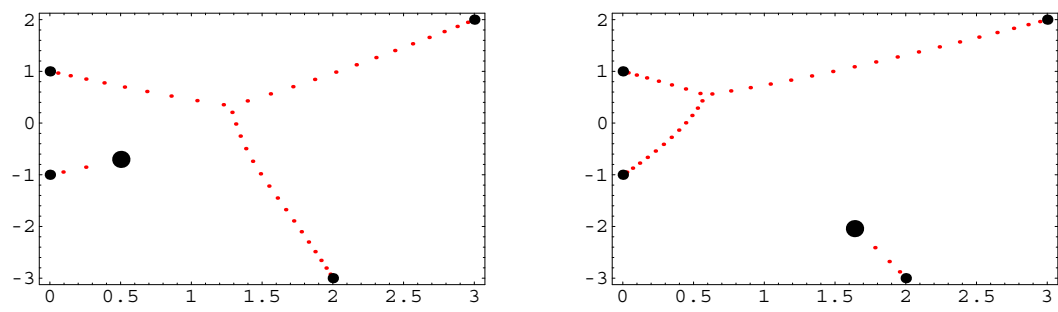

Fig. 6: Examples of $\mu_{Q}$ 's for $T=\left(z^{2}+1\right)(z+2 I-3)(z-3 I-2) \frac{d^{3}}{d z^{3}}$

Consider the following multi-parameter spectral problem. For a given non-negative integer $n$ find all polynomials $V(z)$ of degree at most $r$ such that the equation

$$
T(p(z))+V(z) p(z)=0,
$$

has a polynomial solution $p(z)$ of degree $n$. (Classically, $p(z)$ is called a Stieltjes polynomial and $V(z)$ is called a Van Vleck polynomial.)

Proposition 4 Under the above assumptions for any sufficiently large $n$ there exist exactly $\left(\begin{array}{c}n+r \\ r\end{array}\right)$ degree $n$ Stieltjes polynomials $p_{n, j}(z)$ and corresponding Van Vleck polynomials $V_{n, j}(z)$.

Proposition 5 If a sequence $\left\{\widetilde{V}_{n, j_{n}}(z)\right\}, n=1, \ldots$, of scaled Van Vleck polynomials converges to some polynomial $\widetilde{V}(z)$ then the sequence of finite measures $\mu_{n, j}$ of the corresponding family of eigenpolynomials $\left\{p_{n, j_{n}}(z)\right\}$ converges to a measure $\mu_{\widetilde{V}}$ satisfying the properties:

a) $\operatorname{supp} \mu_{\widetilde{V}}$ is a forest of curvilinear trees;

b) the union of the leaves of $\operatorname{supp} \mu_{\widetilde{V}}$ coincides with the union of all zeros of $Q_{k}(z)$ and those of $\widetilde{V}(z)$.

c) $\operatorname{supp} \mu_{\widetilde{V}}$ is straightened out by the transformation given by

$$
\int_{a}^{z} \frac{\widetilde{V}(z) \mathrm{d} z}{Q_{k}(z)}
$$

Explanations to Fig. 6 and 7. In Fig. 6 we give two examples of different Van Vleck polynomials $V(z)$ and the corresponding Stieltjes polynomials $p(z)$. The average size dots are the 4 roots of the polynomial $Q(z)=\left(z^{2}+1\right)(z+2 I-3)(z-3 I-2)$, the 
unique large dot is the only root of $V(z)$ (which is linear in this case). Small dots show the roots of $p(z)$. In Fig. 7 we show the union of all roots of $p(z)$ of degree 25 for the same problem.

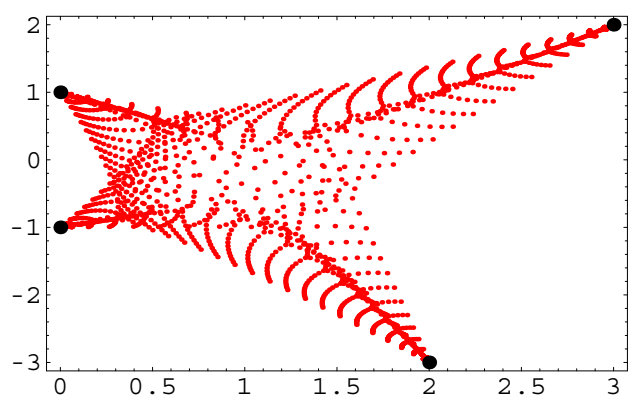

Fig. 7: Union of $\mu_{Q}$ 's for the above $T$

\section{Schrödinger operator with polynomial potential}

This section is based on $[7,8]$. Consider the operator $\mathcal{H}=-\frac{d^{2}}{d z^{2}}+P(z)$ where $P(z)=z^{2 l}+\sum_{i=0}^{2 l-1} a_{i} z^{i}$ is a monic polynomial of even degree with real coefficients. It is well-known that the classical spectral problem

$$
\mathcal{H}(y)=\lambda y
$$

where $y$ belongs to $L_{2}(\mathbb{R})$ has a discrete and simple spectrum $0<\lambda_{0}<\lambda_{1}<\lambda_{2}<\ldots<\lambda_{n}<\ldots$ Denote by $\phi_{0}(z), \phi_{1}(z), \ldots, \phi_{n}(z), \ldots$ the sequence of the corresponding eigenfunctions. These eigenfunctions are real entire functions of order $l+1$ and $\phi_{n}(z)$ has exactly $n$ real zeros. Set $\psi_{n}(z)=\phi_{n}\left(\sqrt[2 l]{\lambda_{n}} z\right)$ which we call the scaled $n$-th eigenfunction.

The Stokes graph of any complex polynomial $P(z)$ is the following object. Each root of $P(z)$ is called a turning point. A (local) Stokes line of $P(z)$ is a maximal segment of the real analytic curve containing at most two turning points (finite or infinite) which solves the equation:

$$
\begin{aligned}
\Re \xi_{z_{0}}(z) & =0 \quad \text { where } \\
\xi_{z_{0}}(z) & =\int_{z_{0}}^{z} \sqrt{P(u)} \mathrm{d} u=0,
\end{aligned}
$$

with respect to $z$, where $z_{0}$ is one of the turning points of $P(z)$. The Stokes graph $S T_{P}$ of the polynomial $P(z)$ is the union of all its local Stokes curves. A local Stokes line connecting two finite turning points, i.e. two roots of $P(z)$ is called short. (The Stokes graph $S T(P)$ of a generic $P(z)$ has no short Stokes lines.)

Proposition 6 For a given positive integer $l$ the Stokes graph $S T\left(z^{2 l}-1\right)$ consists of

1) $l$ short Stokes lines for $l$ odd and $l-1$ short Stokes lines for l even connecting all pairs of the roots of $z^{2 l}-1$ which are symmetric w.r.t the imaginary axis;

2) for $l$ odd each root of $z^{2 l}-1$ is connected by 2 infinite Stokes lines to $\infty$. More exactly, the 2 infinite Stokes lines passing through the root $e^{\frac{\pi i k}{l}}$, $k=0, \ldots, 2 l-1$ are tangent at $\infty$ to the Stokes rays having the nearest slope to $\frac{\pi i k}{l}$;

3) for $l$ even each root of $z^{2 l}-1$ except for $\pm i$ is connected to $\infty$ by 2 infinite Stokes lines with the same property as above. The roots $\pm i$ have 3 infinite Stokes lines each.

Theorem 5 For any monic polynomial $P_{\mathbb{C}}(z)$ of even degree the sequence of meromorphic functions $\left\{\mathcal{C}_{n}(z)\right\}=\left\{\frac{\psi_{n}^{\prime}(z)}{n \psi_{n}(z)}\right\}$ converges to $\mathcal{C}(z)=$ $-K_{l} \sqrt{z^{2 l}-1}$ uniformly on any compact set lying in the domain $\mathbb{C} \backslash U C_{l}$, where $K_{l}=\frac{\sqrt{\pi} \Gamma\left(\frac{3 l+1}{2 l}\right)}{\Gamma\left(\frac{2 l+1}{2 l}\right)}$. (Here by $-\sqrt{z^{2 l}-1}$ we mean the branch which is negative for positive $z>1$. Also $U C_{l}$ is a certain subset of local Stokes lines marked by bold on Fig. 8.)
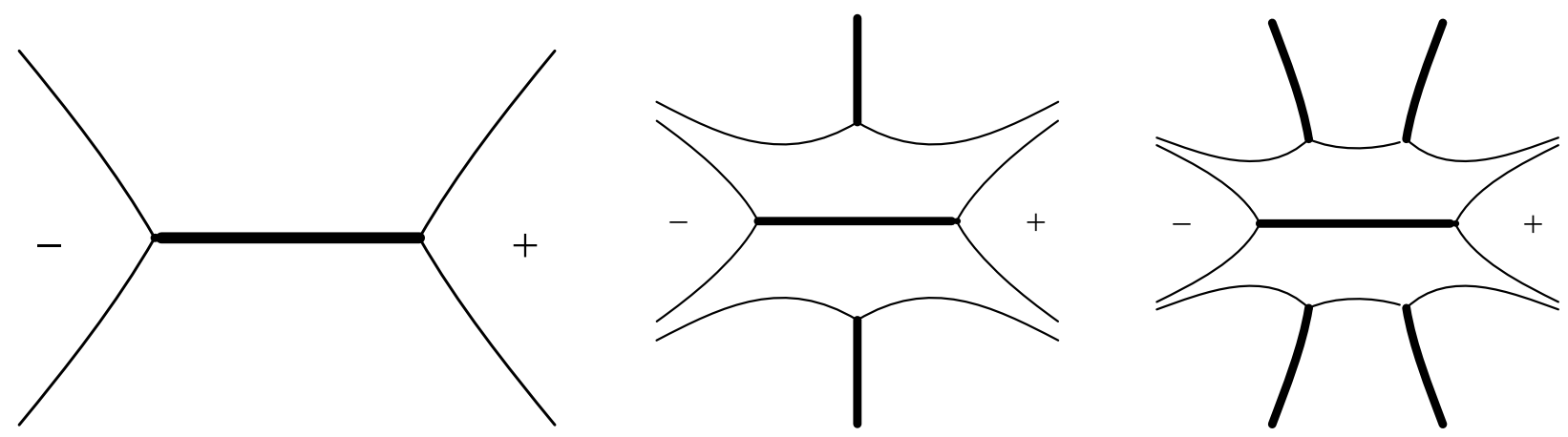

Fig. 8: Stokes lines of $z^{2 l}-1$ for $l=1,2,3$ 


\section{Finite recurrences}

This section is based on [4]. Consider a finite recurrence of length $(k+1)$ given by

$$
p_{n+1}(z)=Q_{1}(z) p_{n}(z)+\ldots+Q_{k}(z) p_{n-k+1}(z),
$$

with polynomial or rational coefficients

$\left\{Q_{1}(z), \ldots, Q_{k}(z)\right\}$ uniquely determined by the initial $k$-tuple $\left\{p_{0}(z), \ldots, p_{k}(z)\right\}$.

Theorem 6 There exists a finite subset $\Theta \subset \mathbb{C}$ depending on the initial $k$-tuple and a curve $\Sigma$ depending on the recurrence such that the asymptotic ratio $\Psi(z)=\lim _{n \rightarrow \infty} \frac{p_{n+1}(z)}{p_{n}(z)}$ exists and satisfies the symbol equation

$$
\Psi^{k}(z)=Q_{1}(z) \Psi^{k-1}(z)+\ldots+Q_{k}(z)
$$

in $\mathbb{C} \backslash(\Sigma \cup \Theta)$. Here $\Sigma$ is the so-called Stokes discriminant of $(*)$ which is the set of all $z$ for which the equation (*) has at most two roots with the same and maximal absolute value.

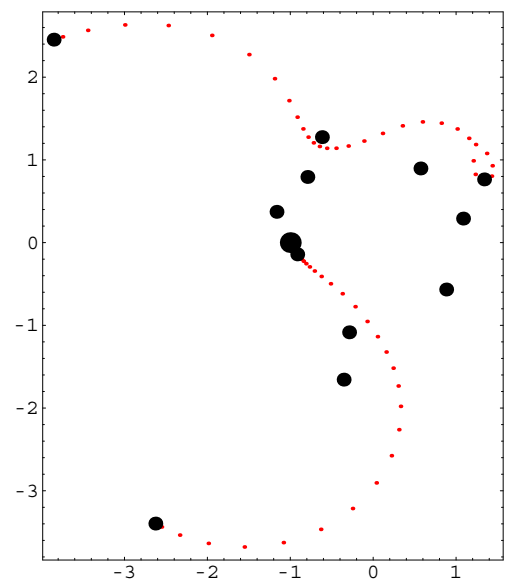

Fig. 9: Zeros of $p_{31}(z)$ satisfying the recurrence relation $(z+1) p_{n}(z)=\left(z^{2}+1\right) p_{n-1}(z)+(z-5 I) p_{n-2}(z)+\left(z^{3}-\right.$ $1-I) p_{n-3}(z)$

\section{Acknowledgement}

I want to thank my coauthors T. Bergkvist, J. Borcea, R. Bøgvad, A. Eremenko, A. Gabrielov, G. Masson, H. Rullgård for the pleasure of working with them and for the numerous insights and results we obtained together. I want to thank the organizers of the miniconference 'Analytic and Algebraic Methods in Quantum Mechanics, V' for the financial support and a great pleasure of visiting Prague in May 2009, where these results were presented.

\section{References}

[1] Bergkvist, T.: On asymptotics of polynomial eigenfunctions for exactly-solvable differential operators, J. Approx. Theory 149(2), (2007), 151-187.

[2] Berqkvist, T., Rullgård, H.: On polynomial eigenfunctions for a class of differential operators, Math. Res. Lett., 9 (2002), 153-171.

[3] Berqkvist, T., Rullgård, H., Shapiro, B.: On Bochner-Krall orthogonal polynomial systems, Math. Scand., 94 (2004), 148-154.

[4] Borcea, J., Bøgvad, R., Shapiro, B.: On rational approximation of algebraic functions, $A d v$. Math. 204 (2006), 448-480.

[5] Borcea, J., Shapiro, B.: Root asymptotics of spectral polynomials for the Lamé operator, Comm. Math. Phys, 282 (2008), 323-337.

[6] Borcea, J., Bøgvad, R., Shapiro, B.: Homogenized spectral pencils for exactly solvable operators: asymptotics of polynomial eigenfunctions, Publ. RIMS, 45 (2009), 525-568.

[7] Gabrielov, A., Eremenko, A., Shapiro, B.: Zeros of eigenfunctions of some anharmonic oscillators, Annales de l'Institut Fourier, 58(2) (2008), 603-624.

[8] Gabrielov, A., Eremenko, A., Shapiro, B.: High energy eigenfunctions of one-dimensional Schrödinger operators with polynomial potentials, Comput. Methods Funct. Theory, 8(2) (2008), 513-529.

[9] Holst, T., Shapiro, B.: On higher Heine-Stieltjes polynomials, to appear in Isr. J. Math.

[10] Masson, G., Shapiro, B.: On polynomial eigenfunctions of a hypergeometric-type operator, $E x$ per. Math., 10 (2001), 609-618.

[11] Shapiro, B.: Algebro-geometric aspects of HeineStieltjes theory, submitted.

[12] Shapiro, B., Takemura, K., Tater, M.: On spectral polynomials of the Heun equation. II, submitted.

Prof. Boris Shapiro

E-mail: shapiro@math.su.se

Department of Mathematics

Stockholm University

SE-106 91, Stockholm, Sweden 